\title{
Reliable Gossip Zone for Real-Time Communications in Wireless Sensor Networks
}

\author{
Bijun Li, Ki-Il Kim, Member, KIMICS
}

\begin{abstract}
Gossip is a well-known protocol which was proposed to implement broadcast service with a high reliability in an arbitrarily connected network of sensor nodes. The probabilistic techniques employed in gossip have been used to address many challenges which are caused by flooding in wireless sensor networks (WSNs). However, very little work has yet been done on real-time wireless sensor networks which require not only highly reliable packets reception but also strict time constraint of each packet. Moreover, the unique energy constraining feature of sensor makes existing solutions unsuitable. Combined with unreliable links, redundant messages overhead in real-time wireless sensor networks is a new challenging issue. In this paper, we introduce a Reliable Gossip Zone, a novel finetailored mechanism for real-time wireless sensor networks with unreliable wireless links and low packet redundancy. The key idea is the proposed forwarding probability algorithm, which makes forwarding decisions after the realtime flooding zone is set. Evaluation shows that as an oracle broadcast service design, our mechanism achieves significantly less message overhead than traditional flooding and gossip protocols.
\end{abstract}

Index Terms - Wireless Sensor Networks, Routing Protocol, Gossip, Real-Time

\section{INTRODUCTION}

IN recent decade, Wireless Sensor Networks (WSNs) have already been widely used for many long-term applications such as military surveillance [1], infrastructure protection [2] and scientific exploration [3]. Since recent wireless sensor technology is becoming more robust and multimedia hardware is getting more miniaturized, WSNS have been provided with multimedia capabilities, which allow new type of real-time multimedia applications such as traffic control and intrusion detection [4]. Therefore, these new missioncritical applications require efficient real-time routing protocols in terms of timeliness, reliability, and energy efficiency.

Broadcasting is an important data communication paradigm in WSNs [5] when a sensor node has to send a

\footnotetext{
Manuscript received March 14, 2011; revised April 1, 2011; accepted April 15, 2011.

The authors are with the Department of Informatics, Engineering Research Institute, Gyeongsang National University, Jinju, 660-701, Korea (Tel: +82-55-772-1373, Fax: +82-55-772-1370, Email: kikim@ gnu.ac.kr). Ki-Il Kim is corresponding author.
}

piece of information to all network nodes. In real-time WSNs, reliable broadcasting is crucial for obtaining good real-time multimedia quality as WSNs exhibit high packet loss rate due to several reasons such as interference, collision route failure, and congestion etc.

Flooding, considered as the simplest solution for supporting broadcast, where each node is required to forward every packet it receives, however, leads to the data implosion and overlapping problems [6], which in turn deplete energy at the sensor nodes, cause degradation of performance and shorten the lifetime of networks. Furthermore, flooding continuous multimedia data leads to severe broadcast collision and channel contention which degrades overall reliability and scalability.

In contrast, probabilistic broadcast approaches, broadly called gossip, offer a simple alternative to deterministic approaches, which avoid the broadcast storm problem and minimize redundant communications. The existing gossiping protocols such as [7], [8], [9], and [10], are predominantly static in nature, that require the gossip probability to be pre- configured, and cannot adapt to the changing topology or the changing application requirements. In this case, nodes in the network are required to forward packets with the same pre-configured probability, which is less than 1 . In order to achieve both high reliability and low packet redundancy, the forwarding probability $p_{\text {gossip }}$ should be chosen correctly. However, it's a difficult problem to choose the correct value of $p_{\text {gossip. }}$. The correct value of $p_{\text {gossip }}$ closely depends on the topology of network, and application requirements. In actual situation, sensor nodes always obtain higher $p_{\text {gossip }}$ than necessary, leading to increased overhead. Moreover, even if $p_{\text {gossip }}$ is chosen correctly at the beginning, it may turn to be unsuitable since topology of network changing or resource consumption.

Correspondingly, the proposed scheme in this paper belongs to another category of gossip-based broadcasting protocols - the adaptive gossip approach. In the proposed scheme, each node calculates individual gossip probability based on a certain algorithm to adapt to network topology and different application requirements. Instead of global $p_{\text {gossip }}$ value, the adaptive $p_{\text {gossip }}$ value at each node guarantees reliability even under node failures and wireless losses. Moreover, the adaptive $p_{\text {gossip }}$ in this scheme keeps low redundant packets received by sink, which achieves energy efficiency eventually.

In addition to decreasing the level of packets redundancy while keeping a high reliability, time 
constraint introduced by real-time communication requires restricting the gossip service within a carefully selected flooding zone between source and sink, which is composed of interconnected nodes. This feature guarantees not only timeliness in real-time WSNs but also energy efficiency [11].

The rest of this paper is organized as follows: Related works are presented in Section II. Section III defines the network model and initialization. Section IV introduces our main design of algorithm, followed by its performance evaluation in Section V. Section VI concludes the paper and shows future work.

\section{RELATED WORKS}

Gossip-based broadcasting protocols generally include two approaches: sender-based and receiver-based. Using ender-based gossip protocol, when a node receives a packet, it selects a subset of its neighbors to forward it. On the other hand, receiver-based gossip protocol makes every node randomly decides whether it forwards the packet or not. For dynamic-topology networks, the former one is considered to be unsuitable since a node does not know its neighbors conditions and make a decision on which node should be in the forwarding subnet in advance.

As an important category for WSNs, the receiver-based gossip protocols have been extensively studied in the literature. Aimed at improving data implosion problem, Smart Gossip [12] adaptively determines the forwarding probability at individual node based on previous knowledge and network topology, to forward received messages.

Haas et al. [7] developed four kinds of schemes for enhancing the static gossip approach, including the basic scheme called Gossip1, and other improved versions: Gossip2 (two-threshold scheme), Gossip3 (preventing premature gossip death scheme), Gossip3 (retries scheme) and Gossip4 (zones scheme).

In [13], gossip probability is proposed to be adaptive to the Energy Map - the information about the amount of energy available at each part of the network, to perform energy-aware broadcasting in WSNs.

Neighbor-aware gossip-based broadcasting scheme [14] makes nodes forward control messages with dynamic probability to decrease the number of redundant messages and thereby improve energy efficiency. By using a novel probability table and adjustment mechanism based on neighbor conditions, nodes can obtain a suitable gossip probability. Furthermore, this work considers some special cases of uneven sensor deployment such as that contains nodes with a low degree.

However, none of these gossip-based broadcasting solutions meets the timeliness requirement of real-time WSNs.

On the other hand, flooding-based routing protocol has been used to broadcast data packets within a carefully pre- defined zone in real-time multimedia WSNs [15]. The proposed flooding zone control protocol monitors the incoming multimedia packets to detect performance deterioration and change the size of flooding zone (increase/decrease) accordingly. This flooding zone scheme meets some application requirements of real-time multimedia WSNs such as timeliness and reliability, however, flooding-based routing protocol lacks of solution for message overhead, which degrades the performance of energy efficiency.

\section{PRELIMINARIES}

This section defines the network model and introduces the initialization process of proposed real-time flooding zone.

\section{A. Network Model}

Suppose there is a wireless sensor network which includes 100 nodes in grid topology, as it is shown in Figure 1. In this simple network, all nodes have a predetermined radio range that each one is able to communicate with 8 of its neighbors, and the links are shown as solid lines. A node can transmit the packet at any time after receives it. Formally, the network can be denoted by a graph $\mathrm{G}=(\mathrm{V}, \mathrm{E})$ where $\mathrm{V}$ is the complete set of 100 nodes in the network and $\mathrm{E}$ is the set of directed edges between each pair of nodes.

To implement the proposed protocol, all nodes are marked with a sequence number from 1 to 100 , and assume that node 12 is a source which sends packets to sink node 67 .

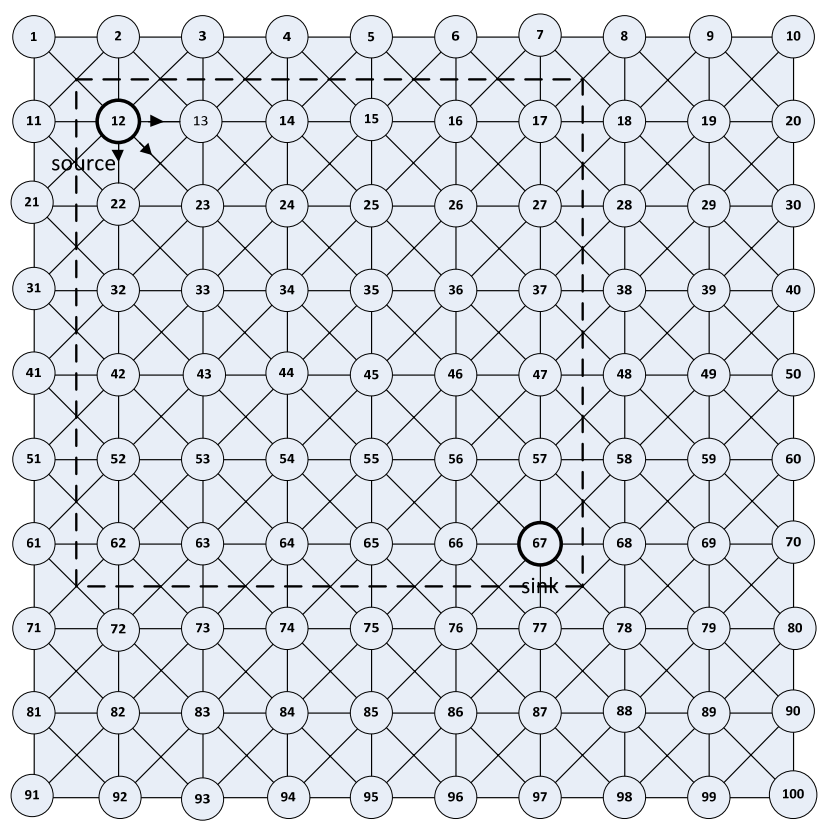

Fig.1. Grid topology of 100 nodes. 
The area surrounded by dashed line is the so-called real-time flooding zone which includes all useful nodes that forward the packets from source node to sink. In different scenarios, this area is set carefully to meet the timeliness requirements of real-time applications and keep the message overhead on a relatively low level. As the very first step of the proposed scheme, the key point lies in how to select the optimal set of nodes for subsequent gossip communication between source node and sink.

\section{B. Initialization}

Suppose the source nodes have 100 packets to be sent throughout the whole network to sink. In order to meet the timeliness requirement of real-time application, and decrease the redundant packets reception rate at the same time, a Real-Time Flooding Zone (RTFZ) should be initialized before the transmission starts.

The initialization a RTFZ involves a two-way handshake process [11]. The process starts by broadcasting an initialization message (Init-msg) from the source node to sink. The Init-msg carries a hopcount value which increases by 1 after it is forwarded one time by an intermediate node. Moreover, the timeliness requirement of the specific real-time multimedia application is also added to this Init-msg as the TTL (Time-To-Live) field to help set the zone under real-time constraint. Once it receives an Init-msg, the intermediate node marks itself with the lowest hopcount value of all Init-msg it received as a Hop-ID which indicates the hop distance from the source node. After sink receives multiple copies of Init-msg from different paths with different hop-count value (Hop-ID), it records and sorts all received Hop-ID values in a table (HopDepth table).

There are several pre-defined policies for intermediate nodes to forward Init-msg as follow:

1. An intermediate node forwards all Init-msg it received except those ones with the same Hop-ID, which are recognized to be duplicated. Each node records the number of upstream nodes it receives Init-msg from.

2. When an intermediate node receives an Init-msg which already expires, it will discard this Init-msg silently, and marks that upstream node as unavailable.

Before sending back an acknowledgment message (Ack-msg) to source node, sink selects a suitable HopDepth value from the Hop-ID table. The Hop-ID table illustrates the status of multiple paths in the network, that how many hops they need for delivering the Init-msg to sink without expiring. Sink chooses the maximum value of all Hop-ID values which it received before deadline, and sends it back to the available last hops as the suitable HopDepth in an Ack-msg. The required packets reception rate $\tau$ is sent back to source node as well within the Ack-msg.
The acknowledgement process employs unicast communication rather than broadcasting, to make this initialization of RTFZ more efficient and accurate. Unicasting policies are shown as follow:

1. Sink unicasts Ack-msg to all available last hops which have sent Init-msg to it within deadline.

2. After receives the Ack-msg, each intermediate node records the lowest hop-count value of all Ack-msg it received as an Ack-ID, at the meantime, the number of downstream nodes that it received Ack-msg from is also recorded.

3. Each intermediate node unicasts Ack-msg to all its available upstream nodes until the source node, which have sent Init-msg to it without expiring.

During the exchange of the Init-msg and the Ack-msg, intermediate nodes learn their relative hop distance from both source and sink nodes by Hop-ID and Ack-ID. Intermediate nodes decide whether to join the RTFZ or not depend on the HopDepth value chosen by sink. If the sum of distances of an intermediate node from both source and sink nodes is less than or equal to the chosen HopDepth, then the node joins the RTFZ area. This process is calculated as follow:

$$
\text { HopDepth } \geq H o p-I D+\text { Ack }-I D
$$

Once an intermediate node decides to join the RTFZ, it continues unicasting Ack-msg to its upstream nodes; if not, it discards Ack-msg silently. After the RTFZ is initialized, the source node starts gossip-based real-time multimedia session by broadcasting the data packets only in the defined real-time flooding zone. Sink node resizes the area of RTFZ only if the timeliness constraint cannot be satisfied with the current hop distance by re-unicasting a newly selected HopDepth, as shown in Figure 2.

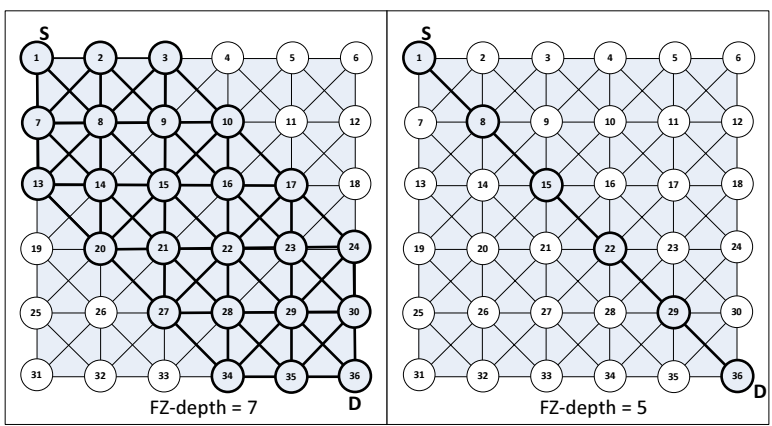

Fig. 2. Different initialized RTFZ sizes.

\section{ALGORITHM DESCRIPTION}

In this section, the proposed gossip-based broadcasting scheme is organized as three phases: 


\section{A. Confirming upstream and downstream nodes}

After the real-time flooding zone is set, all nodes involved in this zone have known the required packets reception rate $\tau$ and the HopDepth chosen by sink. For each node, the first step of calculating the individual gossip probability is to figure out the initialized forwarding probability $P_{i n i}$. For instance, $\tau=90 \%$ implies that the reliability requirement of the application expects sink to receive at least 90 out of 100 packets sent out from the source node. However, if all intermediate nodes forward the packets with a forwarding probability equals to $90 \%$, the final packets reception rate at sink must be less than $90 \%$. From [12], we learn that it's necessary to translate this $\tau$ to a per-hop forwarding probability, $P_{i n i}$. In other words, if each node in the RTFZ forwards packets with a probability as $P_{i n i}$, then sink will receive packets at the packets reception rate it expected. In this case, this $P_{i n i}$ could be considered as the maximum forwarding probability of each node. $P_{i n i}$ is calculated using this equation:

$$
\tau=\left(P_{\text {ini }}\right)^{\text {HopDepth-1 }}
$$

With this notion of initialized forwarding probability for each hop in the RTFZ, a node with a single upstream node requires more than $P_{i n i}$. However, when a node has $n_{p}$ upstream nodes, the probability $P_{r e q}$ that at least one of them transmits with must exceed the per-hop initialized probability. In order to calculate this $P_{\text {req }}$, all intermediate nodes pass Hello messages to their upstream nodes after the RTFZ is set, to notice each upstream node that how many downstream nodes it has, which implies the importance of each upstream node. Then, by using the equation shown below, a node collects the information from all its downstream nodes, and selects the minimum value $n_{p}^{\prime}$ which indicates this downstream node has the highest reception requirement of all downstream nodes $n_{c}$.

$$
n_{p}^{\prime}=\min \left(n_{p i}\right), i=\left(1,2, \ldots, n_{c}\right)
$$

After $n_{p}^{\prime}$ is selected by one node, the following equation dictates the choice of gossip probability:

$$
\left(1-P_{r e q}\right)^{n_{p}^{\prime}}=\left(1-P_{i n i}\right)
$$

In this equation, $\left(1-P_{\text {req }}\right)$ denotes the probability that a particular upstream node does not forward the packet. Assume that all upstream nodes of one intermediate node independently decide whether to forward or not, the probability of all $n_{p}^{\prime}$ upstream nodes choose not to forward could be calculated as $\left(1-P_{r e q}\right)^{n_{p}^{\prime}}$. If this value equals to the failure probability $\left(1-P_{i n i}\right)$, it means the required forwarding probability $P_{r e q}$ is the lower threshold for one upstream node.

\section{B. Calculating suitable gossip probability}

The proposed gossip-based broadcasting scheme is an adaptive gossip protocol that adaptively calculates the suitable gossip probability for an particular upstream node based on relationships between the this node and its downstream nodes. Since the higher threshold and lower threshold of gossip probability have already been found, each node in the RTFZ needs a method to calculate individual gossip probability before forwards the first packet it received.
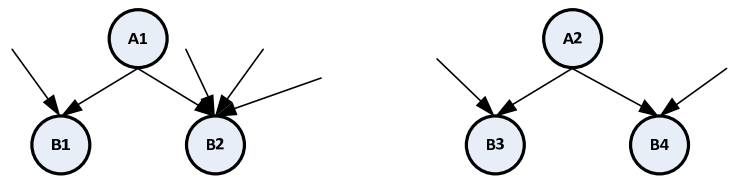

Fig. 3. Example of different neighbor conditions with the same number of downstream nodes

In this phase, the work is to figure out a method to measure the importance of one upstream node to all its downstream nodes. Here we use neighbor conditions of each node to compute a corrected value that denotes the importance of one upstream node. The numbers of downstream nodes of node $\mathrm{A} 1$ and $\mathrm{A} 2$ are the same in Figure 3. According to equation (2) and equation (3), node A1 and A2 have the same $P_{r e q}$ as the lower threshold of gossip probability. However, one downstream node B2 of node A1 has more upstream nodes than B4 of A2, which implies that as an upstream node, A2 plays more important role to its downstream nodes than A1. As a result, A2 is considered to have higher gossip probability. The following equations are used to compute true gossip probability of a node, where $n_{c}$ is the number of downstream nodes of one node, $n_{c}$ is the minimum number of upstream nodes, $\Delta P$ is the corrected value for individual node.

$$
\Delta P=\left(P_{i n i}-P_{r e p}\right) \cdot \frac{1}{n_{p}^{\prime}} \cdot \frac{n_{c} / \sum_{j=1}^{n_{c}} n_{p j}}{\sum_{i=1}^{n_{c}} \frac{1}{n_{p i}} / n_{c}}
$$

For all nodes in the RTFZ, the value of $P_{r e q}$ depends on the downstream node which has the minimum number of 
upstream nodes $-n_{p}^{\prime}$. The gap between $P_{i n i}$ and $P_{r e q}$, $\left(P_{i n i}-P_{r e p}\right)$ can be thought as the 'risk' of 'putting trust on this number of upstream nodes', so $\left(P_{i n i}-P_{r e p}\right) \bullet 1 / n_{p}^{\prime}$ means each upstream node shares this 'risk', to guarantee the reliability. The last part of this equation indicates a coefficient to evaluate the importance of one upstream node to all its downstream nodes. The numerator $n_{c} / \sum_{j=1}^{n_{c}} n_{p j}$ is the ratio of links which come from an upstream node, to the sum of links which go to all downstream nodes. It is considered to be a weight value to inflect the importance of one node to its downstream nodes. The denominator $\sum_{i=1}^{n_{c}} \frac{1}{n_{p i}} / n_{c}$ is the average value of that for each downstream node, the ratio of the link comes from the particular upstream node to the number of all upstream nodes it has. For this correction value, the more close to 1 , the more similar neighbor condition each downstream node has. If it equals to 1 , it means that all downstream nodes have the same number of $n_{p}$, and require the same $P_{\text {req }}$ value; if it is less than 1 , it means one or more downstream nodes have more than $n_{p}^{\prime}$ upstream nodes, which require lower forwarding probability than $P_{r e q}$. In this case, upstream node takes it into account and decreases $P_{r e q}$ accordingly to lighten the message overhead.

Once this correction value of probability $\Delta P$ is calculated, each upstream node uses the equation below to generate the true gossip probability:

$$
P_{g s p}=P_{r e q}+\Delta P
$$

\section{Handling first-hop nodes}

This phase attempts to identify the sensor node at a particular location of the real-time flooding zone. In this proposed scheme, the particular sensor nodes refer to the first-hop nodes from the source nodes, which have the greatest impact to the whole network if transmission failure happens. Since the influence of transmission failure decreases proportionally from the source node to sink, to afford reliability and stability of the proposed protocol, it is necessary to set the probability of the firsthop nodes equals to 1 , to avoid the packets from dying out at the very beginning.

\section{PERFORMANCE EVALUATION}

To confirm performance enhancement, the proposed scheme is compared with the traditional flooding and a static gossip protocol Gossip1 [7]. These three protocols are implemented on C language. For simulations, 100 nodes are distributed in an area with grid topology as shown in Figure 1. The distance between nodes with consecutive numbers is set as $170 \mathrm{~m}$, which leads to the diagonal distance of two nodes to be about $250 \mathrm{~m}$, very close to the default radio radius of one node.

The evaluation is first set in a perfect network environment without link and node errors, and then link and node failure modules are added to the previous network setting. All simulations are supposed to show the enhancement of proposed scheme in timeliness, reliability and energy efficiency by reducing overhead generated by redundant packets.

\section{A. Performance without errors}

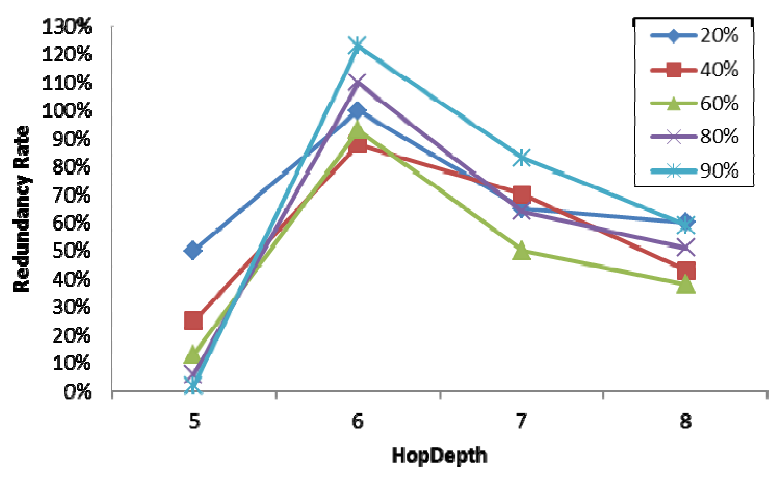

Fig. 4. Performance of proposed scheme without errors

Without errors, the proposed scheme performs well in all scenarios as shown in Figure 4. For four realtime flooding zones set by different HopDepth, the proposed scheme not only guarantees reliability of packet transmission, but also keeps low-level redundancy of duplicate packets, which is the critical issue in existing flooding and gossip protocols. In Figure 4, when HopDepth is equal to 5, which indicates that there's only one path is available from source node to sink in this scenario, the number of redundant packets received by sink decreases inversely as the growth of required packet reception rate. Due to the fact that there's only one path transferring packets in this case, the result is reasonable and acceptable. When the HopDepth value is equal to a number from 6 to 8 , the number of redundant packets increases proportionally according to growth of required packet reception rate. Since there's no error on both nodes and links, and forwarding policy is supposed that duplicate packets will be discarded once they have received by one node, the zone which has less HopDepth transfers more packets due to a higher forwarding probability calculated by proposed algorithm. However, in some other scenarios which include definite errors on both nodes and links, this condition will be changed. 


\section{B. Comparison without errors}

Integrated with all three kinds of protocols, Figure 5 shows that without errors, the proposed protocol performs much better than others, which is meant to be more suitable for a real-time flooding zone. Comparing with others, the proposed protocol has less redundant packets in all scenarios, while Gossip1 leads to proportionally several times more redundant packets, and Flooding performs the worst with the most redundant packets. The monotone decreasing tendency of Flooding attributes to the same amount of packets received by sink but different requirements of reliability in each scenario.

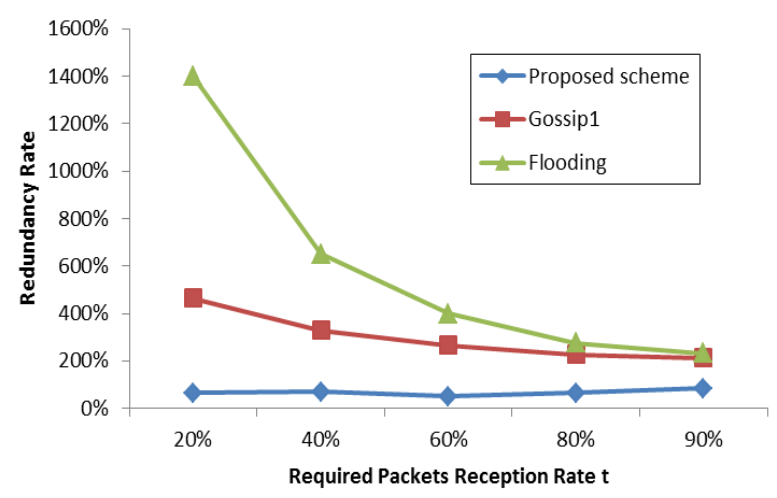

Fig. 5. Comparison of three protocols without errors

\section{Comparison at defined HopDepth with errors}

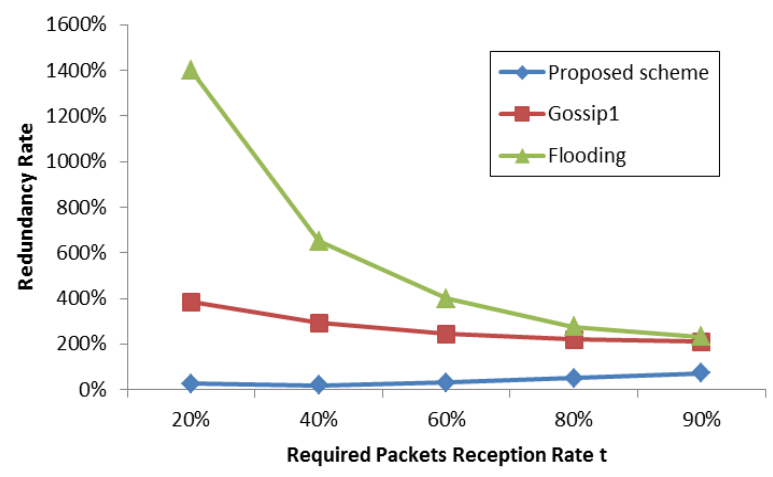

Fig. 6. Comparison of three protocols with errors at defined HopDepth

In Figure 6, the proposed protocol denotes that although some packets are lost during transmission due to the link and node failures, it still guarantees the reliability required by sink with relatively low redundancy. Accordingly, Gossip1 also has a little amount of packets lost, but the number of redundant packets is still great. Flooding has almost the same amount of packets received by sink as without errors.

\section{Comparison at defined packets reception rate with errors}

The forth scenario is set with errors and required packets reception rate as $80 \%$, in Figure 7, Flooding shows a huge amount of redundant packets, while Gossip1 performs similarly to it. This is because both of them employ static forwarding probabilities when forward packets, which couldn't adapt the situation of each node. Instead of static one, the proposed protocol uses an adaptive probabilistic technique to calculate a suitable forwarding probability on each node, which leads to less redundancy at sink.

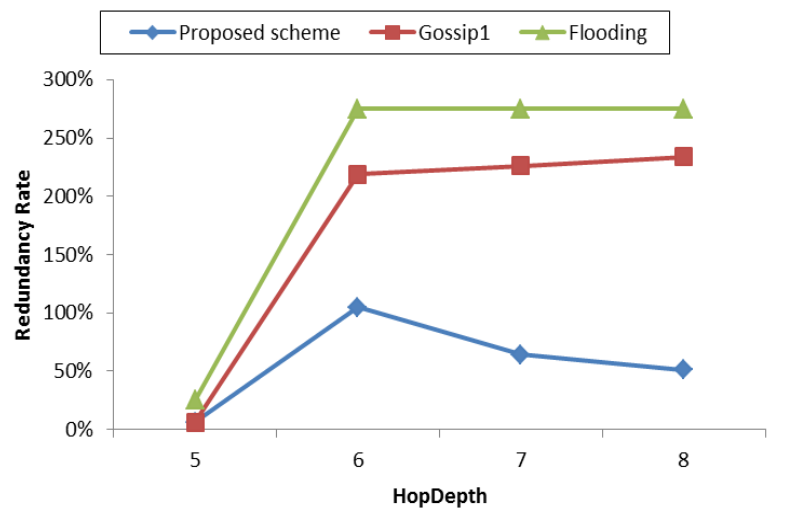

Fig. 7. Comparison of three protocols with errors and defined packets reception rate

\section{VI.CONCLUSION AND FUTURE WORK}

This work presents the proposed gossip-based broadcasting protocol for real-time multimedia sensor networks. It involves two phases: a real-time flooding zone initialization that set to guarantee timeliness requirement of real-time applications, and an adaptive gossip-based forwarding algorithm which considers both maintaining the required reliability and energy efficiency by reducing redundant packets to lighten the message overhead. Extensive simulations and evaluation show that the proposed protocol approaches much better performance with lower redundancy rate than traditional Flooding and Gossip1. The reliable gossip zone protocol proposed in this paper is designed for the scenario where the energy of sensor nodes is relatively high. In the future, we shall extend this work into the scenario where all sensor nodes have different energy conditions, and each node should take its energy condition into account when forwarding packets.

\section{ACKNOWLEDGMENT}

This research was supported by the MKE (The Ministry of Knowledge Economy), Korea, under the ITRC(Information Technology Research Center) support program supervised by the NIPA(National IT Industry Promotion Agency (NIPA-2011-C1090-1031-0007). 


\section{REFERENCES}

[1] T. He, S. Krishnamurthy, L. Luo, T. Yan, L. Gu, R. Stoleru, G. Zhou, Q. Cao, P. Vicaire, J. A. Stankovic, T. F. Abdelzaher, J. Hui, and B. Krogh, "VigilNet: An Integrated Sensor Network System for Energy-Efficient Surveillance," ACM Transactions on Sensor Networks, Vol. 2, February 2006, pp. 1-38.

[2] N. Xu, S. Rangwala, K. K. Chintalapudi, D. Ganesan, A. Broad, R. Govindan, and D. Estrin, "A Wireless Sensor Network for Structural Monitoring," in Proc. ACM SenSys, November 2004.

[3] L. Selavo, A. Wood, Q. Cao, T. Sookoor, H. Liu, A. Srinivasan, Y. Wu, W. Kang, J. Stankovic, D. Young, and J. Porter, "LUSTER: Wireless Sensor Network for Environmental Research," in Proc. ACM SenSys, November 2007.

[4] S. Mishra, M. Reisslein, and G. Xue, "A Survey of Multimedia Streaming in Wireless Sensor Networks," IEEE Communications Surveys and Tutorials, Vol. 10, January 2008, pp. 18-39.

[5] M. Heissenb"uttel, T. Braun, M. W"alchli, and T. Bernoulli, "Optimized Stateless Broadcasting in Wireless Multi-hop Networks," in Proc. IEEE INFOCOM, April 2006.

[6] Sze-Yao Ni, Yu-Chee Tseng, Yuh-Shyan Chen, and Jang-Ping Sheu, "The Broadcast Storm Problem in a Mobile Ad Hoc Network," in Proc. ACM/IEEE MobiCom, August 1999, pp. 151162.

[7] Zygmunt J. Haas, Joseph Y. Halpern, and Li Li, "Gossip-Based Ad Hoc Routing," in Proc. IEEE INFOCOM, June 2002.

[8] R. Chandra, V. Ramasubramanian, and K. P. Birman, "Anonymous Gossip: Improving Multicast Reliability in Mobile Ad-Hoc Networks," in Proc. IEEE ICDCS, April 2001.

[9] P. Levis, N. Patel, D. Culler, and S. Shenker, "Trickle: A SelfRegulating Algorithm for Code Propagation and Maintenance in Wireless Sensor Networks," in Proc. Symposium on Network System Design and Implementation (NSDI), March 2004.

[10] M. Miller, C. Sengul, and I. Gupta, "Exploring the Energy-Latency Tradeoff for Broadcasts in Energy-Saving Sensor Networks," in Proc. IEEE ICDCS, June 2005.

[11] T. Elamsy, and R. El-Marakby, "Flooding Zone Initialization Protocol (FZIP): Enabling Efficient Multimedia Diffusion for Multi-hop Wireless Networks," in Proc. IEEE Symposium on Computers and Communications (ISCC), July 2008, pp. 10561061.

[12] P. Kyasanur, R. R. Choudhury, and I. Gupta., "Smart Gossip: An Adaptive Gossip-based Broadcasting Service for Sensor Networks," in Proc. IEEE MASS, October 2006.

[13] Max do V. Machado, Raquel A.F. Mini, and Antonio A.F. Loureiro, "Gossiping using the Energy Map in Wireless Sensor Networks," in Proc. ACM MSWiM, October 2007.

[14] T-Ch Huang, Y-P Lin, and Lung Tang, "Netighbor-Aware GossipBased Broadcasting Scheme for Wireless Sensor Networks," in Proc. IEEE International Conference on Communications and Mobile Computing (CMC), April 2010.

[15] T. Elamsy, and R. El-Marakby, "Flooding Zone Control Protocol (FZCP): Enhancing the Reliability of Real-Time Multimedia Delivery in WSNs," in Proc. IEEE International Symposium on Signal Processing and Information Technology (ISSPIT), December 2009.

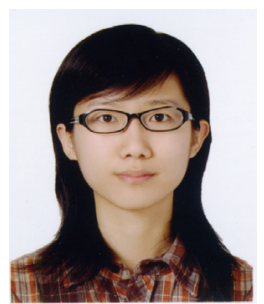

Bijun Li is currently working toward Ph.D in Gyeongsang National University, Jinju, Korea. Her research interests include wireless sensor networks, and routing protocol.

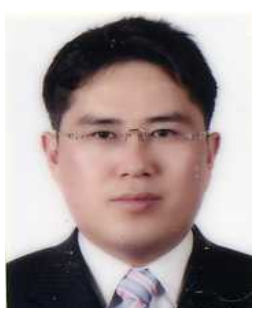

Ki-Il Kim received the M.S. and Ph.D. degrees in computer science from the ChungNam National University, Daejeon, Korea, in 2002 and 2005 , respectively. He is currently with the Department of Informatics at Gyeongsang National University. His research interests include routing for MANET, QoS in wireless network, multicast, and sensor networks. 